\title{
Exercício físico e fibromialgia
}

\author{
Roberta Chiden Bueno ${ }^{a}$, Merquiene Freitas Abreu ${ }^{a}$, Gabriel Natan Pires ${ }^{\mathrm{b}, \mathrm{c}}$, Diego Roger-Silva ${ }^{\mathrm{a}, \mathrm{c}}$ \\ ${ }^{a}$ Especialista em Atividade Física Adaptada, \\ Universidade Gama Filho - UGF, Rio de Janeiro, RJ, Brasil

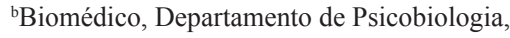 \\ Universidade Federal de São Paulo - UNIFESP, São Paulo, SP, Brasil \\ ${ }^{\mathrm{c} C e n t r o ~ d e ~ E s t u d o s ~ T e o ́ r i c o s ~ A p l i c a d o s ~ a ̀ ~ S a u ́ d e, ~ U n i v e r s i d a d e ~ I b i r a p u e r a ~-~ U N I b, ~ S a ̃ o ~ P a u l o, ~ S P, ~ B r a s i l ~}$
}

\begin{abstract}
Resumo: A síndrome da fibromialgia é uma doença reumática não inflamatória que acomete principalmente as mulheres, sendo que $93 \%$ dos atingidos são de etnia branca. A síndrome da fibromialgia pode ser classificada como primária, quando não há relação com outra patologia, ou secundária, quando esta se encontra associada com alguma patologia. O paciente fibromiálgico precisa receber tratamento multidisciplinar, ou seja, áreas distintas devem trabalhar em conjunto para que haja uma melhora nos sintomas. Dentre os sintomas observados na fibromialgia, o mais comum é a dor crônica e difusa. A literatura apresenta referências que apontam os efeitos e benefícios do exercício físico como forma de tratamento não-medicamentoso para os portadores da síndrome da fibromialgia. Dentre os exercícios físicos estão a atividade aeróbica, alongamentos e treinamento de força, entre outros. O objetivo desta revisão é apresentar as formas pelas quais o exercício físico pode ser útil ao paciente fibromiálgico, sobretudo no que tange a melhora dos seus sintomas.
\end{abstract}

Palavras-chave: Fibromialgia, Tratamento, Exercício.

\section{Physical exercise and fibromyalgia}

\begin{abstract}
Fibromyalgic syndrome is a non-inflammatory rheumatic disease which affects primarily Caucasian women. Fibromyalgic syndrome can be classified as primary, when there is no other associated pathology; or secondary, when it is diagnosed related to some other pathology. The fibromyalgic patient needs to receive multidisciplinary treatment and different areas should work together to promote the improvement of symptoms. The most common classical symptom of this disease is the chronic and diffuse pain. The specialized literature presents several works that point out the effects and benefits of physical exercise as a non-pharmacological treatment for patients with fibromyalgic syndrome. Aerobic activity, stretching and strength training are among the physical exercises. Thus, this review aimed to highlight the several ways physical exercise can be useful to the fibromyalgic patient, especially concerning the improvement of symptoms.
\end{abstract}

Keywords: Fibromyalgia, Therapeutics, Exercise.

\section{Introdução}

A Síndrome da Fibromialgia (SFM) é caracterizada por dores musculoesqueléticas crônicas e difusas originadas pelo sistema nervoso central, sendo uma doença reumática não-inflamatória (SANTOS; KRUEL, 2009; CHAITOW et al., 2002). Considerada de etiologia desconhecida, a fibromialgia apresenta diagnóstico clínico polimorfo, devendo ser avaliada por uma cuidadosa anamnese e exame físico detalhado, tendo o seu tratamento direcionado ao controle dos sintomas (KUCHINAD et al., 2007; WOOD et al., 2007; PROVENZA et al., 2004).

Devido à dificuldade do diagnóstico dessa síndrome, para a classificação da fibromialgia os critérios utilizados são baseados pelo Colégio

Autor para correspondência: Gabriel Natan Pires, Departamento de Psicobiologia, Universidade Federal de São Paulo, Rua Napoleão de Barros, 925, Vila Clementino, CEP 04024-002, São Paulo, SP, Brasil, e-mail: gnspires@gmail.com Recebido em 18/3/2011; $1^{\text {a }}$ Revisão em 17/8/2011; Aceito em 14/12/2011. 
Americano de Reumatologia (American College of Rheumatology - ACR). De acordo com estudos realizados por Wolfe et al. (1990) o ACR confirma o diagnóstico de fibromialgia quando o individuo apresentar dor generalizada há pelo menos três meses, combinada com a sensibilidade em onze ou mais dos dezoito pontos sensíveis, sendo esses pontos ou sítios de sensibilidade designados como tender points, palpados em uma pressão de $4 \mathrm{kgf..}$ Recentemente, um método alternativo ao diagnóstico de fibromialgia e independente da avaliação de tender points foi proposto pelo ACR (WOLFE et al., 2010). Todavia, esse método ainda necessita de maiores avaliaçóes para tornar-se clinicamente aplicável, de modo que o diagnóstico clássico descrito por Wolfe et al. (1990) continua sendo a principal estratégia diagnóstica à fibromialgia.

Os nove pontos bilaterais dolorosos (tender points) correspondem a inserçóes tendíneas ao osso ou a ventres musculares e estão localizados na inserção dos músculos occipitais, entre os processos transversos de C5 a C7 (paravertebrais cervicais), borda superior do trapézio, supra-espinhais, segunda junção condrocostral (nas superfícies das costelas superiores), epicôndilos laterais dos cotovelos, glúteos médios, trocânteres maiores dos fêmures e interlinhas mediais dos joelhos (Figura 1).

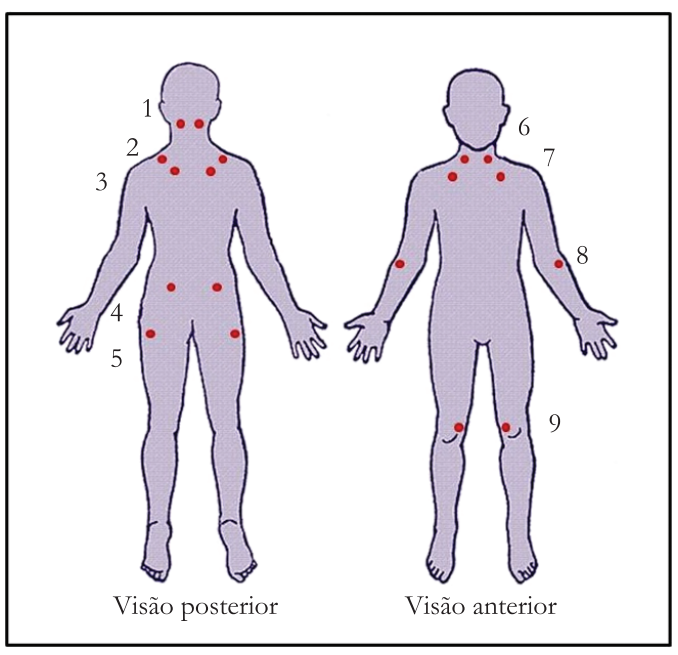

Figura 1. Localização dos 9 pares de tender points. 1) inserção dos músculos occipitais; 2) borda superior do trapézio; 3) musculos supra-espinhais; 4) gluteo médios; 5) trocânteres maiores dos fêmures; 6) entre os processos transversos de $\mathrm{C} 5 \mathrm{a}$ C7; 7) Segunda junção condrocostal; 8) epicôndilos laterais dos cotovelos; 9) interlinhas mediais dos joelhos.
Associam-se à SFM diversos sintomas frequentes como fadiga, alteraçóes de humor, cefaléias, rigidez matinal, distúrbios de sono, entre outros (WOLFE et al., 1990; HOFFMAN; DUKES, 2008). Esses sintomas afetam negativamente a rotina dos pacientes fibromiálgicos, acarretando limitaçôes funcionais e disfunções psicológicas, interferindo no desempenho das atividades diárias, no trabalho e consequentemente na qualidade de vida (MARTINEZ et al., 1992; WHITE et al., 1999).

Os sintomas típicos da fibromialgia pioram com o frio, umidade, tensão e inatividade e são aliviados por calor, atividade moderada e relaxamento. A dor muscular é considerada o principal sintoma da SFM. Geralmente é descrita pelos pacientes fibromiálgicos como uma sensação de queimação. Essa dor pode mudar de localização sendo mais intensa nas partes do corpo com maior frequência de uso (BATES; HANSON, 1998; BALSAMO; SIMÃO, 2005). A fadiga também é um sintoma bastante frequente, sendo mais perceptível pela manhã e ao final do dia, e estando associada aos distúrbios de sono (SNIDER, 2000). As alteraçôes psicológicas e comportamentais também afetam grande parte do paciente fibromiálgico. Neste âmbito, torna-se comum a presença de transtornos de humor, como depressão, transtornos de ansiedade e irritabilidade. Todavia, mais estudos se fazem necessários para que se possa atribuir relaçóes de causa e conseqüência entre fibromialgia e as condiçôes psicológicas citadas (KNOPLICH, 2003).

Segundo Cavalcante et al. (2006) a fibromialgia acomete $5 \%$ da populaçáo em geral e $10 \%$ da população brasileira na faixa etária de 30 a 60 anos, podendo também ocorrer em crianças e idosos. Considerando todos os acometidos por essa patologia, 93\% são da etnia branca. Além disso, a síndrome em questão afeta prioritariamente as mulheres, apresentando-se em uma freqüência de até 15 mulheres para cada homem. Nos atendimentos de clínica médica essa frequência é em torno de 5\%, nos pacientes hospitalizados $7,5 \%$ e nos serviços de clínicas reumatológicas a SFM é detectada entre $14 \%$ dos atendimentos.

Maurizio e Rogers, (1997) classificam a fibromialgia em duas categorias, sendo elas, primária ou secundária. A fibromialgia primária apresenta quadro clínico da doença sem haver relação a outras patologias. Já a secundária ocorre quando o paciente fibromiálgico possui este quadro de modo concomitantemente ou responsivo a outra patologia.

Conforme Sabbag et al. (2007), a SFM deve receber tratamento multidisciplinar composto por farmacoterapia, terapia psicossocial e 
atividade física. $\mathrm{O}$ tratamento medicamentoso é constituido por anti-inflamatórios, relaxantes musculares, analgésicos e antidepressivos tricíclicos (PROVENZA et al., 2004). Na fibromialgia os anti-inflamatórios não são muito eficazes, porém auxiliam no controle da dor quando em associação com outros medicamentos. Contudo, apresentam efeitos colaterais possíveis, em especial quando são usados de forma contínua. Os antidepressivos tricíclicos trazem benefícios em curto prazo, pois possuem ação analgésica indireta, promovem aumento na biodisponibilidade de neurotransmissores específicos, como serotonina, dopamina e norepinefrina. Além disso, essa medicação não causa dependência e não possui efeito narcótico (LEITÃO, 1995).

O exercício físico é um dos métodos de tratamento que apresenta excelentes resultados no controle da SMF (VALIM, 2001). Trata-se de um meio de promover saúde em diversos aspectos, sendo capaz de diminuir a dor e os sintomas da fibromialgia (VALIM, 2006). Em contrapartida, a falta de exercícios físicos em pacientes com SFM ocasiona um declínio na função neuromuscular, na resistência muscular, na velocidade de contraçáo dos músculos e na função cardiorrespiratória, interferindo totalmente no desempenho funcional e em atividades cotidianas como, por exemplo, caminhar, subir escadas, entre outras (FISHER, 2004).

O presente ensaio tem por objetivo apresentar estudos relacionados ao exercício físico aplicado a pacientes com SFM, relatando os diversos aspectos da síndrome. Deve-se levar em consideração que a fibromialgia é uma doença de difícil diagnóstico, que acomete um considerável número de pessoas. A mesma afeta diretamente a qualidade de vida e atividades rotineiras desenvolvidas pelos pacientes com SFM. Deste modo, é de grande interesse estudos que ajudem na maior compreensão desta síndrome, assim como estudos que objetivem esclarecer modos de tratamento, como o exercício físico, no contexto da SFM.

\section{Exercício físico no contexto da fibromialgia}

Os pacientes com fibromialgia em geral têm um nível de controle cardiovascular autonômico menor que a média (REYES DEL PASO et al., 2011) e o consumo de oxigênio é menor do que em pacientes não fibromiálgicos (BATES; HUDSON, 1998; DINLER et al., 2009). Como resultado, eles tendem facilmente a ficar mal condicionados, o que diminui sua eficiência cardiovascular e circulação periférica (BATES; HANSON, 1998). A atividade física apresenta papel fundamental em relação à qualidade de vida, pois a prática proporciona boa disposição física e mental, aliviando as tensôes diárias e aumentando a expectativa de vida (NAHAS, 2003; RIBEIRO; FUSCO, 2005). A prática de exercícios proporciona sensação de bem estar e de autocontrole, apresentando efeito analgésico, relacionado às dores características da patologia em questão (SABBAG et al., 2000; MARQUES et al., 2002).

Um programa de exercícios físicos adequados e executados de maneira regular melhora a coordenação motora e outras capacidades físicas importantes para a realização das atividades diárias em pessoas genéricas e especialmente em pacientes fibromiálgicos (JONES; LIPTAN, 2009; RIBEIRO; FUSCO, 2005; ORTEGA et al., 2009). Ainda, estabelece uma postura adequada e auxilia no controle da massa corporal, da ansiedade e da condição cardiovascular (RIBEIRO; FUSCO, 2005). Chaitow et al. (2002) observou que os exercícios aumentaram a liberação de hormônios como endorfinas, gerando uma sensação de conforto, alívio da dor e encorajamento psicológico, proporcionando tanto melhora no condicionamento físico quanto dos sintomas nociceptivos.

Inicialmente os programas de exercícios, podem ocasionar um aumento dos sintomas, principalmente dor e fadiga, contudo, com a continuidade das atividades, esses desconfortos tendem a diminuir. Os benefícios começam a aparecer entre a oitava e décima semana após o inicio dos exercícios efetuados e continuam aumentando até a vigésima semana, se sobrepondo ao desconforto inicial (VALIM et al., 2003; PFRIMER, 2008). Assim, a prática regular de exercícios físicos pode ser adotada como uma abordagem de otimização do tratamento da fibromialgia, promovendo redução da dor e do impacto dos outros sintomas, restabelecendo a capacidade física, mantendo a funcionalidade e promovendo melhora na qualidade de vida (MARTINEZ et al., 1998).

Assim como a prescrição de medicamentos deve conter dose, duração e intervalo específicos, a prescrição do exercício ao paciente fibromiálgico também deve ser detalhada, sobretudo quando utilizada como estratégia terapêutica não-medicamentosa. Os profissionais responsáveis precisam manter certos cuidados e estarem atentos em relação à intensidade e execução de exercícios, preferencialmente iniciando com cargas leves e progredindo de acordo com as necessidades e evolução de cada indivíduo. Para a prescrição individual ser adequada, é importante considerar 
as preferências do paciente, co-morbidades, uso de medicamentos, capacidade funcional e, se possível, avaliação ergométrica (VALIM et al., 2003).

\section{Efeitos de diferentes modalidades de exercício}

Atualmente existe uma variedade de programas de exercícios destinados a pacientes com SFM. Contudo, devemos considerar que alguns exercícios apresentam melhores resultados do que outros, no controle dos sintomas da doença.

\subsection{Exercícios aeróbicos}

Dentre os diferentes programas de exercícios os aeróbicos, as atividades de baixa intensidade e de longa duração, têm sido destacados como eficientes no manejo do paciente fibromiálgico (MANIDI; MICHEL, 2001). De acordo com McArdle et al. (2003) o treinamento aeróbico induz adaptaçôes em várias capacidades funcionais relacionadas com o transporte e utilização de oxigênio e com adaptaçóes metabólicas no músculo esquelético. Segundo Valim et al. (2003) os exercícios aeróbicos apresentam vantagens sobre exercícios de alongamento no tratamento da SFM, uma vez que o treino aeróbico promove mudanças neuroendócrinas como o aumento na liberação da serotonina e norepinefrina, resultando na melhora do humor, efeito náo observado no alongamento. A aplicaçáo do exercício aeróbico de baixo impacto em pacientes com SFM deve ser com gradativo aumento de carga e intensidade até 65-70\% da frequência cardíaca máxima (VALIM et al., 2003). Alguns estudos com exercícios aeróbicos em solo demonstraram, que as pacientes obtiveram efeito antidepressivo e relaxante e diminuição da dor nos tender points em $74 \%$ das pacientes com fibromialgia (SIM; ADAMS, 1999).

\subsection{Alongamento}

Conforme Routi et et al. (2000) exercícios de alongamento são exercícios de flexibilidade que são realizados com o intuito de aumentar a amplitude do movimento de uma articulação ou de uma série de articulaçóes. A caminhada associada aos exercícios de flexibilidade, em um período de três meses, mostrou melhora em 35\% dos indivíduos com SFM submetidos à intervenção (MANNERKORPI; IVERSEN, 2003). Assim, os alongamentos musculares podem gerar impacto positivo na SFM, promovendo melhora em parâmetros associados à fibromialgia, como a baixa qualidade de sono e a rigidez matinal (RICHARDS; SCOTT, 2002).

\subsection{Treinamento de força}

Segundo Fleck e Kraemer (1999) o termo treinamento de força pode ser descrito como um tipo de exercício físico que requer que os músculos se movam ou tentem se mover contra uma determinada resistência. Neste caso a resistência normalmente é representada por algum tipo de equipamento, seja em forma de máquinas ou pesos livres. Hakkinen et al. (2001), em um estudo no qual avaliaram o treinamento de força progressiva em paciente com SFM, demonstraram uma diminuição no impacto da doença sobre o sistema neuromuscular, sobre a percepção dos sintomas e sobre a capacidade funcional. Assim, este tipo de exercício pode ser utilizado seguramente no tratamento da SFM. Outro estudo demonstrou resultados significativos em exercícios resistidos praticados por portadores de SFM durante oito semanas, duas vezes por semana, executando três séries de 10 repetiçóes, com cargas de $60 \%$ e $70 \%$ de uma repetição máxima. Os resultados demonstraram que esse método de exercícios melhorou significativamente os sintomas da doença, em especial a fadiga e melhora na qualidade do sono e humor (JENTOFT et al., 2001).

\subsection{Atividade física aquática}

Um estudo seguindo os critérios do American College of Sports Medicine (ACSM) conduzido com pacientes do sexo feminino, entre 18 e 65 anos e com diagnóstico clínico de fibromialgia avaliou as pacientes através de uma anamnese contendo dados demográficos e clínicos, por questionário de qualidade de vida (SF36) e avaliou o efeito da aplicação de atividades físicas realizadas em piscina aquecida sobre os sintomas da fibromialgia. Tais atividades consistiram de 10 minutos de alongamento, 40 minutos de treinamento aeróbico e 10 minutos de relaxamento, três vezes por semana em piscina aquecida num período de 16 semanas. A fisioterapia aquática em piscina aquecida foi eficaz como parte do tratamento para mulheres com fibromialgia, melhorando diversos aspectos, como qualidade de vida, capacidade funcional, dores, limitaçôes por aspectos físicos, aspectos sociais e emocionais (MAGALHÂES et al., 2008). Existem relatos dos benefícios dos exercícios na água pré-aquecida no tratamento da SFM, pois há uma melhora na qualidade do sono e na qualidade de vida (MANNERKORPI et al., 2002). Conforme 
Dull (2001) as atividades em água aquecida são extremamente benéficas aos portadores da SFM. Devido à sustentação contínua proporcionada por ela, consegue-se diminuir a sobrecarga óssea de maneira ideal, promovendo relaxamento muscular. Bates e Hanson (1998) explicam que exercícios em piscina aquecida são benéficos devido a uma ausência de força concêntrica. Segundo os autores, os movimentos são mais lentos por serem realizados na água fazendo com que diminua as chances de micro traumas na musculatura. Deste modo as atividades aquáticas induzem aumento tanto na tolerância ao exercício quanto no nível de resistência, acarretando uma melhora geral no nível de condicionamento e fazendo com que a intensidade dos sintomas diminua.

\subsection{Reeducação Postural Global}

Pasqual et al. (1994) conduziram estudo descritivo com a intençáo de avaliar o efeito dos exercícios de alongamento muscular usando o método da reeducação postural global (RPG) em indivíduos com fibromialgia. Neste estudo foram avaliadas e tratadas 20 pacientes do sexo feminino com idade entre 23 e 61 anos. As seções de exercícios variaram de 3 a 11. Foram realizados exercícios de alongamentos das cadeias encurtadas. O nível de dor foi avaliado segundo os seguintes critérios: ótimo (ausência de dor), bom (melhora importante e dor esporadicamente), regular (queixas e dor cíclicas) e ruim (ausência de melhora). Dentre as participantes deste estudo, 65\% classificaram a melhora como ótima e boa, $25 \%$ tiveram melhora cíclica em períodos com dor ausente. Somente $10 \%$ afirmaram não ter tido melhora alguma.

\section{Considerações gerais sobre prescrição de exercício}

A rotina adequada de treinamento de aptidão cardiovascular contribui para melhorar a qualidade do sono e consequentemente, os sintomas da SFM (MOLDOFSKY, 2002). Assim, exercícios físicos devem ser prescritos e realizados de forma programada e organizada, uma vez que são considerados promotores na redução dos sintomas da SFM (MARQUES et al., 2002). Adams e Sim (2005) enfatizam a importância de respeitar o princípio da individualidade biológica, caracterizando as diferenças de cada indivíduo. Ressaltam ainda que a melhor modalidade de exercício é aquela na qual o paciente sente mais prazer durante e após a realização, seja ela relacionada a atividades aquáticas, caminhada ou outra modalidade.
O Grupo de Apoio à Pacientes com Fibromialgia da Universidade Federal de São Paulo (UNIFESP) recomenda, em relação às modalidades de exercícios, a prática de exercícios de alongamento, no mínimo duas vezes por semana. Na musculação, a frequência pode ser de duas a três vezes por semana, com série de oito a doze repetiçóes. O ACSM confirma a importância da realização das atividades físicas diariamente, indicando de cinco a sete vezes por semana, com duração de pelo menos trinta minutos de intensidade moderada. Exercícios físicos aeróbicos como caminhar, andar de bicicleta, correr e fazer hidroginástica, quando realizados com intensidade de leve a moderada contribuem para uma melhor função cardiorrespiratória sendo considerados como os mais indicados para a para a SFM.

\section{Conclusão}

No contexto geral em relação à SFM, constatou-se que os diferentes tipos de tratamento são realizados em conjunto. Existe a integração de diversas terapias, envolvendo distintas áreas como a Educação Física, Fisioterapia, Farmacologia entre outras. Todas essas áreas devem atuar de maneira integrada, em busca da diminuiçấo da dor e outros sintomas.

Os sintomas que desencadeiam a SFM afetam diretamente a vida dos portadores. Além do desconforto e da dor generalizada pelo corpo, todo o individuo tem um declínio em sua rotina, afetando a vida profissional e pessoal do paciente fibromiálgico. Os profissionais que atendem esse público devem ser cuidadosos na elaboraçáo e manutenção do tratamento, ficando alerta em relação às necessidades e individualidades de todos os pacientes. Os indivíduos com fibromialgia precisam estar cientes dos benefícios que as terapias podem trazer, fazendo com que possam controlar os sintomas aumentando a qualidade de vida.

Existem diferentes programas de exercícios físicos. Com base nos estudos avaliados, pode-se perceber que cada um contribui de alguma forma para o tratamento da fibromialgia. É importante levar em consideração as preferências dos pacientes em relação às atividades propostas, pois o exercício praticado precisa ser prazeroso ao paciente tanto durante quanto após a execução. Alguns estudos descrevem que o exercício aeróbico é superior no tratamento da SFM, sendo mais eficaz nos resultados obtidos quando comparado a outras modalidades. Porém os exercícios de alongamento também obtiveram resultados positivos em relação à diminuição dos sintomas. Os exercícios de força com cargas adequadas, também 
foram considerados um método seguro que contribui no tratamento da doença.

O exercício físico deve ser tomado como um promotor do alívio da dor, gerando bem estar e contribuindo de forma significativa para a qualidade de vida de pacientes com fibromialgia. Pode-se constatar que a prescrição de um programa de exercícios quando bem elaborado e também supervisionado, ajuda os portadores de fibromialgia a alcançar um aumento gradual do condicionamento físico, da flexibilidade e da habilidade funcional.

\section{Referências}

ADAMS, N.; SIM, J. Rehabilitation Approaches in Fibromyalgia. Disability and Rehabilitation, London, v. 12 , n. 27 , p. 711-723, June 2005. http://dx.doi. org/10.1080/09638280400014709

BALSAMO, S.; SIMÁO, R. Treinamento de Força: para Osteoporose, Fibromialgia, Diabetes Tipo 2, Artrite Reumatoide e Envelhecimento. São Paulo: Phorte, 2005.

BATES, A.; HANSON, N. Exercícios Aquáticos Terapêuticos. São Paulo: Manole, 1998.

CAVALCANTE, A. B. et al. A prevalência da fibromialgia: uma revisão de literatura. Revista Brasileira de Reumatologia, São Paulo, v. 46, n. 1, p. 40-48, jan./fev. 2006.

CHAITOW, L. et al. Sindrome da Fibromialgia: um guia para o tratamento. Sáo Paulo: Barueri: Manole, 2002.

DINLER, M. et al. Effect of aerobic exercise training on oxygen uptake and kinetics in patients with fibromyalgia. Rheumatology International, Berlin, v. 30, n. 2, p. 281-284, Dec 2009. PMid:19784655. http://dx.doi.org/10.1007/ s00296-009-1126-x

DULL, H. Watsu: exercícios para o corpo na água. São Paulo: Summus, 2001.

FISHER, N. M. Osteoartrite, artrite reumatóide e fibromialgia. In: AMERICAN COLLEGE OF SPORTS MEDICINE (Comp.). Pesquisas do ACSM para Fisiologia do Exercício Clínico: Afecçôes Musculoesqueléticas, Neuromusculares, Neoplásicas, Imunológicas e Hematológicas. Rio de Janeiro: Guanabara Koogan, 2004. cap. 10, p. 125-139.

FLECK, S. J.; KRAEMER, W. Fundamentos do Treinamento de Força Muscular. 2. ed. Porto Alegre: Artes Médicas Sul Ltda., 1999.

HAKKINEN, A. et al. Strength training induced adaptations in neuromuscular function of premenopausal women with fibromyalgia: comparison with healthy women. Annals of the Rheumatic Diseases, London, v. 60, n. 1, p. 21-26, Jan 2001. PMid:11114277 PMCid:1753355. http://dx.doi.org/10.1136/ard.60.1.21

HOFFMAN, D. L.; DUKES, E. M. The health status burden of people with fibromyalgia: a review of studies that assessed health status with the SF- 36 or the SF- 12. International Journal of Clinical Practice, Surrey, v. 62, n. 1, p. 115-126, Jan 2008.
JENTOFT, E. S. et al. Effects of pool-based and land-based aerobic on womwn with fibromyalgia/chronic widespread muscle pain. Arthritis \& Rheumatism, Atlanta, v. 45, n. 1, p. 42-47, Feb 2001. http://dx.doi.org/10.1002/15290131(200102)45:1<42::AID-ANR82>3.0.CO;2-A

JONES, K. D.; LIPTAN, G. L. Exercise interventions in fibromyalgia: clinical applications from the evidence. Rheumatic Disease Clinics of North America, Washington, v. 35, n. 2, p. 373-391, May 2009. PMid:19647149. http:// dx.doi.org/10.1016/j.rdc.2009.05.004

KNOPLICH, J. Enfermidades da Coluna Vertebral: uma visão clínica e fisioterápica. 3. ed. São Paulo: Robe Editorial, 2003.

KUCHINAD, A. et al. Accelerated brain gray matter loss in fibromyalgia patients: premature aging of the brain? Journal of Neuroscience, Baltimore, v. 27, n. 15, p. 4004-4007, Apr 2007.

LEITÃo, A. Clínica de Reabilitação. São Paulo: Atheneu, 1995.

MAGALHÃES, R. et al. Avaliação da qualidade de vida em mulheres com fibromialgia após fisioterapia aquática. Revista da FARN, Natal, v. 7, n. 1, p. 13-27, 2008.

MANIDI, M. J.; MICHEL, J. P. Atividade Física para adultos com mais de 55 anos: quadros clínicos e programas de exercícios. Barueri: Manole, 2001.

MANNERKORPI, K. et al. Six- and 24-month follow-up of pool exercise therapy and education for patients with fibromyalgia. Scandinavian Journal of Rheumatology, Stockholm, v. 31, n. 5, p. 306-310, 2002. PMid:12455823. http://dx.doi.org/10.1080/030097402760375223

MANNERKORPI, K.; IVERSEN, M. D. Physical exercise in fibromyalgia and related syndromes. Best Practice and Research Clinical Haematology, London, v. 17, n. 4, p. 629-647, Aug 2003.

MARQUES, A. et al. A fisioterapia no tratamento de pacientes com fibromialgia: uma revisão de literatura. Revista Brasileira de Reumatologia, São Paulo, v. 42, n. 1, p. 42-48, jan./fev. 2002.

MARTINEZ, J. E. et al. Fibromialgia: aspectos clínicos e socioeconômicos. Revista Brasileira de Reumatologia, São Paulo, v. 32, n. 5, p. 225-230, 1992.

MARTINEZ, J. E. et al. Análise crítica de parâmetro de qualidade de vida em fibromialgia. Acta Fisiátrica, São Paulo, v. 5, n. 2, p. 116-120, ago. 1998.

MAURIZIO, S.; ROGERS, J. Recognizing and treating fibromyalgia. Nurse Practitioner, Springhouse, v. 22, n. 12, p. 18-33, Dec 1997.

McARDLE, W. et al. Fisiologia do Exercício: energia, nutriçáo e desempenho humano. 5. ed. Rio de Janeiro: Guanabara Koogan, 2003.

MOLDOFSKY, $\mathrm{H}$. Management of sleep disorders in fibromyalgia. Rheumatic Disease Clinics of North America, Washington, v. 28, n. 2, p. 353-365, May 2002.

NAHAS, M. V. Atividade física, saúde e qualidade de vida: conceitos e sugestôes para um estilo de vida ativo. 3. ed. Londrina: Midiograf, 2003. 
ORTEGA, E. et al. Exercise in fibromyalgia and related inflammatory disorders: known effects and unknown chances. Exercise Immunology Review, Champaign, v. 15, p. 42-65, 2009. PMid:19957871.

PASQUAL, A. et al. Alongamento muscular em pacientes com fibromialgia a partir de um trabalho de reeducação postural global (RPG). Revista Brasileira de Reumatologia, São Paulo, v. 34, n. 5, p. 232-234, set./out. 1994.

PFRIMER, L. Atividade física adaptada à osteoartrite, fibromialgia e dor miofascial. In: TEIXEIRA, L. Atividade física adaptada e saúde. São Paulo: Phorte, 2008. p. 161-163. PROVENZA, J. R. et al. Projeto Diretrizes Fibromialgia. São Paulo: Sociedade Brasileira de Reumatologia, 2004.

REYES DEL PASO, G. A. et al. Autonomic cardiovascular control and responses to experimental pain stimulation in fibromyalgia syndrome. Journal of Psychosomatic Research, Oxford, v. 70, n. 2, p. 125-134, Feb 2011. PMid:21262414. http://dx.doi.org/10.1016/j.jpsychores.2010.09.012

RIBEIRO, K. L.; FUSCO, I. S. Fibromialgia e Atividade Física. Fitness \& Performance Journal, Rio de Janeiro, v. 4, n. 5 p. 280-287, set./out. 2005.

RICHARDS, S. C.; SCOTT, D. L. Prescribed exercise in people with fibromyalgia; parallel group randomized controlled trial. BMJ: British Medical Journal, London, v. 325, p. 185-187, 2002. PMid:12142304 PMCid:117444. http://dx.doi.org/10.1136/bmj.325.7357.185

ROUTI, R. T. et al. Reabilitação aquática. São Paulo: Manole, 2000.

SABBAG, L. et al. Estudo ergométrico de portadores de fibromialgia primaria em programa de treinamento cardiovascular supervisionado. Acta Fisiátrica, São Paulo, v. 7, n. 1, p. 29-34, abr. 2000.

SABBAG, L. et al. Efeitos do condicionamento físico sobre pacientes com fibromialgia. Revista Brasileira de Medicina do Esporte, São Paulo, v. 13, n. 1, p. 6-10, jan./fev. 2007. http://dx.doi.org/10.1590/S1517-86922007000100003

SANTOS, L. C.; KRUEL, L. F. M. Síndrome de Fibromialgia: fisiopatologia, instrumentos de avaliação e efeitos do exercício. Motriz: revista de educacao fisica, Rio Claro, v. 15, n. 2, p. 436-448, abr./jun. 2009.

SIM, J.; ADAMS, N. Physicalandother no pharmacologicalinterventions for fibromyalgia. Best Practice and Research Clinical Rheumatology, London, v. 13, n. 3, p. 507-523, Sept. 1999. http://dx.doi.org/10.1053/ berh.1999.0041

SNIDER, R. K. Tratamento das Doenças do Sistema Músculo Esquelético. Rio de Janeiro: Manole, 2000.

VALIM, V. Estudos dos efeitos do condicionamento aeróbico e do alongamento na fibromialgia. 2001. $117 \mathrm{f}$. Tese (Doutorado em Reumatologia)-Escola Paulista de Medicina, Universidade fedreral de São Paulo, Sáo Paulo, 2001.

VALIM, V. et al. Aerobic Fitness in fibromyalgia. Journal of Rheumatology, Toronto, v. 30, n. 5, p. 1060-1069, 2003.

VALIM, V. Benefícios dos Exercícios Físicos na Fibromialgia. Revista Brasileira Reumatologia, São Paulo, v. 46, n. 1, p. 49-55, 2006.

WHITE, K. P. et al. Comparing selfreported function and work disability in 100 comunity cases of fibromyalgia syndrome versus controls in London, Ontario. Arthritis \& Rheumatism, Atlanta, v. 42, n. 1, p. 76-83, Jan 1999. http:// dx.doi.org/10.1002/1529-0131(199901)42:1<76::AIDANR10>3.0.CO;2-G

WOLFE, F. et al. The American College of Rheumatology 1990. Criteria for the classification of fibromyalgia: Report of the multicenter criteria committee. Arthritis \& Rheumatism, Atlanta, v. 33, n. 2, p. 160-172, Feb 1990.

WOLFE, F. et al. The American College of Rheumatology preliminary diagnostic criteria for fibromyalgia and measurement of symptom severity. Arthritis Care \& Research, New York, v. 62, n. 5, p. 600-610, May 2010. WOOD, P. B. et al. Reduced presynaptic dopamine activity in fibromyalgia syndrome demonstrated with positron emission tomography: a pilot study. Journal of Pain, Philadelphia, v. 8, n. 1, p. 51-58, Jan 2007.

\section{Contribuição dos Autores}

Roberta Chiden Bueno: Concepção do texto, revisão bibliográfica e redação do texto. Merquiene Freitas Breu: Concepção do texto, revisão bibliográfica e redação do texto. Gabriel Natan Pires: Redação do texto, revisão biliográfica, revisão técnica, elaboração da figura. Diego Roger-Silva: Concepção do texto, revisão bibliográfica, redação do texto e revisão técnica. 